\title{
Semiregular Variables: The Character of Variability and Possible Subdivision of the SRc Class
}

\author{
L. S. Kudashkina, I. L. Andronov \\ Astronomical Observatory, Odessa State University, T. G. Shevchenko \\ Park, Odessa 270014, Ukraine
}

\begin{abstract}
It is proposed to split the SRc class into two subclasses with prototype stars PZ Cas and S Per.
\end{abstract}

We use a set of photometric parameters (amplitude, mean amplitude, temporal variability of frequency and amplitude) for the detailed classification of $\mathrm{SR}$ variables. For the periodogram analysis, we have used the computer codes by Andronov (1994).

We studied brightness variations of $\mathrm{PZ}$ Cas during $14 \mathrm{yr}$ (Andronov, Kudashkina, \& Romanenko 1992). The period of this star increased from 801 to $900 \mathrm{~d}$. After this, from 1994 the star had nearly constant brightness, and until 1997 showed small occasional increases of the brightness. An inversion of the asymmetry of PZ Cas with a 18-yr cycle was suggested by using earlier photographic observations (1957-1983) by Kudashkina (1985).

$S$ Per is a SRc-type star with a strong modulation of the amplitude of pulsations. The peak in the periodogram is double: $P_{1}=809.6 \pm 0.2$ and $P_{2}=768$ d $8 \pm 0.3$. Besides that, there is a peak in the periodogram corresponding to the cycle of $16170^{\mathrm{d}} \pm 160^{\mathrm{d}}$. The light curve of $\mathrm{S}$ Per has been regular since JD 2447000 with the period 816 d 8 . Suggesting that the star pulsates in the fundamental mode and using the classical relationships for blackbody and $P-L$ relationship for supergiants (Feast 1980), we have estimated the radius of $S$ Per to be approximately $1400 R_{\odot}$.

The conclusions are: The type SRc can be divided into at least two subtypes: (1) SRca - supergiant stars with multiperiodic pulsations and regular light curves of the Mira-type, sometimes disturbed by the switching mode intervals ( $\mathrm{S}$ Per); and (2) SRcb - supergiant stars with quasi-periodic light curves and intervals of brightness constancy (PZ Cas).

\section{References}

Andronov, I. L. 1994, Odessa Astron. Publ., 7, 49

Andronov, I. L., Kudashkina L. S., \& Romanenko, T. V. 1992, Perem. Zvezdy, $23,1,23$

Feast, M. W. 1980, in Proc. Fifth Eur. Reg. Meet. (Liége: Inst. d'Astrophys.), P.B.1.1

Kudashkina, L. S. 1985, IBVS, No. 2791 